\title{
Tumor-related gene expression levels in pulmonary pleomorphic carcinoma
}

\author{
Takeshi Oyaizu1', Yuji Matsumura²*, Satoru Kobayashi ${ }^{1}$, Tetsu Sado ${ }^{1}$, Hiromi Ishihama ${ }^{1}$ and Masayuki Chida ${ }^{1}$
}

\begin{abstract}
Background: Pulmonary pleomorphic carcinoma (PPC) is a rare type of non-small-cell lung cancer that belongs to the family of sarcomatoid carcinomas and is associated with poor prognosis. We investigated the expressions of tumor-related genes in resected PPC specimens.

Methods: Specimens resected from patients with PPC from July 2006 through April 2012 were investigated. Tumor segments were collected from the specimens by micro-dissection to extract mRNA, then RT-PCR was performed according to Dannenberg's tumor profile method for semi-quantitation of tumor-related gene mRNA. To compare with other types of lung cancer, data from stage-matched adenocarcinoma (AC) and squamous cell carcinoma (SCC) cases in our database were also examined.
\end{abstract}

Results: The gene expression levels of thymidylate synthase were significantly higher in PPC and SCC as compared to the AC specimens $(p<0.001)$. The levels of dihydropyrimidine dehydrogenase and thymidine phosphorylase mRNA in PPC showed a similar tendency to those in SCC, in contrast to AC. Furthermore, the expression level of excision repair cross-complementation group 1 mRNA in PPC specimens was similar to that reported in NSCLC, while the level of vascular endothelial growth factor (VEGF) expression was higher as compared to that reported for colorectal cancer.

Conclusions: Although gene expression of tumor cannot be directly correlated to its sensitivity for anti-cancer drugs, it is likely that PPC tumors are not sensitive to anti-metabolic drugs. Anti-VEGF therapy may be a candidate for PPC, while cisplatin also remains a viable option.

Keywords: Lung cancer, Pleomorphic carcinoma, Gene expression

\section{Background}

A pulmonary pleomorphic carcinoma (PPC) is a rare type of non-small-cell lung cancer (NSCLC) that belongs to the family of sarcomatoid carcinomas [1] and is associated with poor prognosis [2-4]. Most affected patients experience recurrence and die during the first year after surgical intervention [2]. In general, the chemo-sensitivity of PPC is considered to be lower than that of other types of NSCLC, though Kawano et al. conducted a histoculture drug response assay and reported that some anticancer drugs may be effective [3]. Recently, the expression of various tumor-related genes have been reported to correlate to chemo-sensitivity and prognosis in several types of cancer [5-7]. However, few reports have been published

\footnotetext{
* Correspondence: yuji-m@dokkyomed.ac.jp

${ }^{2}$ Department of Cardiovascular and Thoracic Surgery, Dokkyo Koshigaya Hospital, 2-1-50 Minamikoshigaya, Koshigaya, Saitama 343-8555, Japan
} Full list of author information is available at the end of the article regarding tumor-related gene expression levels in PPC. In the present study, we investigated the expressions of tumor-related genes in this rare tumor to establish an appropriate treatment strategy.

\section{Methods}

Resected specimens from patients with PPC who underwent lung resection from July 2006 through April 2012 at our institutions were investigated. Those from patients who received preoperative induction therapy including chemotherapy or chemoradiotherapy were excluded. To compare with other types of lung cancer, 40 stage-matched adenocarcinoma (AC) and 35 squamous cell carcinoma (SCC) cases in our database [8] were also examined. Dokkyo Medical University Hospital Ethics Committee approved this retrospective study (\#24035) and waived the need for patient consent for analysis of the results. 


\section{Determination of tumor-related gene expression}

Excised specimens were thinly sliced, then tumor segments were collected by micro-dissection to extract mRNA. RTPCR was performed according to Danenberg's tumor profile (DTP) method [9] for semi-quantitation of mRNA of tumor-related genes (Response Genetics Inc., New York, USA), including thymidylate synthase (TS), dihydropyrimidine dehydrogenase (DPD), thymidine phosphorylase (TP), excision repair cross-complementation group 1 (ERCC1), vascular endothelial growth factor (VEGF), and topoisomerase-1 (TOPO1). In brief, a laser captured micro-dissection technique was used on $10-\mu \mathrm{m}$ sections with a magnification of $100 x$ to obtain only cancer cells. Next, 1500-2000 round spots $80 \mu \mathrm{m}$ in diameter were dissected for each case, from which total RNA was extracted. Real-time quantitative reverse transcription-PCR assays were performed on those samples using an ABI 7700 and TaqMan Probes. The level of $ß$-actin mRNA was used as a reference gene. Relative gene expression values are expressed as a ratio of PCR products of the gene of interest to that of the internal reference gene $ß$-actin.

\section{Statistics}

Values are shown as the mean \pm SD. Analysis of variance (ANOVA) was used for comparing among 3 groups, then Tukey's post-hoc test was used when significance was found. Differences were considered significant at $P<0.05$, with borderline significance considered at $P<0.10$.

\section{Results}

Among 536 patients who underwent surgery for primary lung cancer at our institution, 10 had PPC (1.9\%). They consisted of 9 men and 1 woman, with a mean age of 64.9 years (range 47-80 years). Patient characteristics are shown in Table 1.

Gene expression levels of the nucleotide-metabolismrelated enzymes TS, DPD, and TP in PPC were compared to those in $\mathrm{AC}$ and $\mathrm{SCC}$ specimens. The relative gene expression value of TS was $5.65 \pm 3.46$ in PPC, $1.59 \pm 0.86$ in $\mathrm{AC}$, and $4.68 \pm 3.71$ in SCC (Fig. 1). That value was significantly higher in PPC and SCC specimens as compared to $\mathrm{AC}(p<0.001)$. In contrast, the relative gene expression value for DPD was $2.49 \pm 1.70$, $2.20 \pm 1.18$, and $1.59 \pm 1.34$, respectively (Fig. 2), which was greater in PPC and AC than in SCC, with borderline significance $(p=0.0649)$. That of TP in the specimens was $14.81 \pm 14.99,9.17 \pm 6.51$, and $14.61 \pm 11.06$, respectively (Fig. 3), with no significant difference between AC and SCC, while that of AC was significantly lower as compared to SCC $(p<0.05)$.

Other tumor-related gene expression levels are shown in Table 2. The relative gene expression values of ERCC1, VEGF, and TOPO1 in the PPC specimens were $1.68 \pm 0.66,8.53 \pm 6.44$, and $2.55 \pm 0.73$, respectively.

\section{Discussion}

PPC is a rare type of tumor and accounted for only $1.9 \%$ of resected lung cancer cases examined in the present study. Although affected patients are known to

Table 1 Patient characteristics

\begin{tabular}{|c|c|c|c|c|c|c|}
\hline Case & Age/Sex & Stage & Chemotherapy & Relapse & & Status \\
\hline \multirow[t]{2}{*}{1} & $70 / \mathrm{M}$ & T2bN1M0 & $(+)$ & Distant & $16 \mathrm{mo}$ & $\overline{\text { Dead }}$ \\
\hline & & & AMR & & & \\
\hline 2 & $75 / M$ & T2bN1M0 & $(-)$ & $(-)$ & $39 \mathrm{mo}$ & Alive \\
\hline \multirow[t]{2}{*}{3} & $57 / M$ & T2aNOMO & $(+)$ & Distant & $42 \mathrm{mo}$ & Alive \\
\hline & & & AMR, CBDCA + PTX & & & \\
\hline 4 & $77 / \mathrm{M}$ & T2bNOMO & $(-)$ & Distant & $9 \mathrm{mo}$ & Dead \\
\hline \multirow[t]{2}{*}{5} & $68 / M$ & T2aNOMO & $(+)$ & $(-)$ & $57 \mathrm{mo}$ & Alive \\
\hline & & & $\mathrm{DTX}+\mathrm{TS} 1$ & & & \\
\hline 6 & $80 / M$ & T3NOMO & $(-)$ & Distant & $57 \mathrm{mo}$ & Alive \\
\hline 7 & $54 / M$ & T3NOMO & $(-)$ & Local $^{a}$ & $2 \mathrm{mo}$ & Dead \\
\hline \multirow[t]{2}{*}{8} & $47 / M$ & T2bNOMO & $(+)$ & Distant & $12 \mathrm{mo}$ & Dead \\
\hline & & & $\mathrm{TS} 1, \mathrm{CDDP}+\mathrm{VNR}, \mathrm{CBDCA}+\mathrm{PTX}$ & & & \\
\hline \multirow[t]{2}{*}{9} & $48 / F$ & T2aN1M1 & $(+)$ & Distant & $14 \mathrm{mo}$ & Dead \\
\hline & & & CBDCA + DTX & & & \\
\hline 10 & $73 / \mathrm{M}$ & T2bNOMO & $(-)$ & $(-)^{\mathrm{b}}$ & $2 \mathrm{mo}$ & Dead \\
\hline
\end{tabular}

Each stage is presented based on the 7th UICC TNM classification

mo months, AMR amrubicin, CBDCA carboplatin, PTX paclitaxel, TS1 tegafur gimeracil oteracil, DTX docetaxel, VNR vinorelbine aincomplete resection

boperative death due to cerebral infarction 


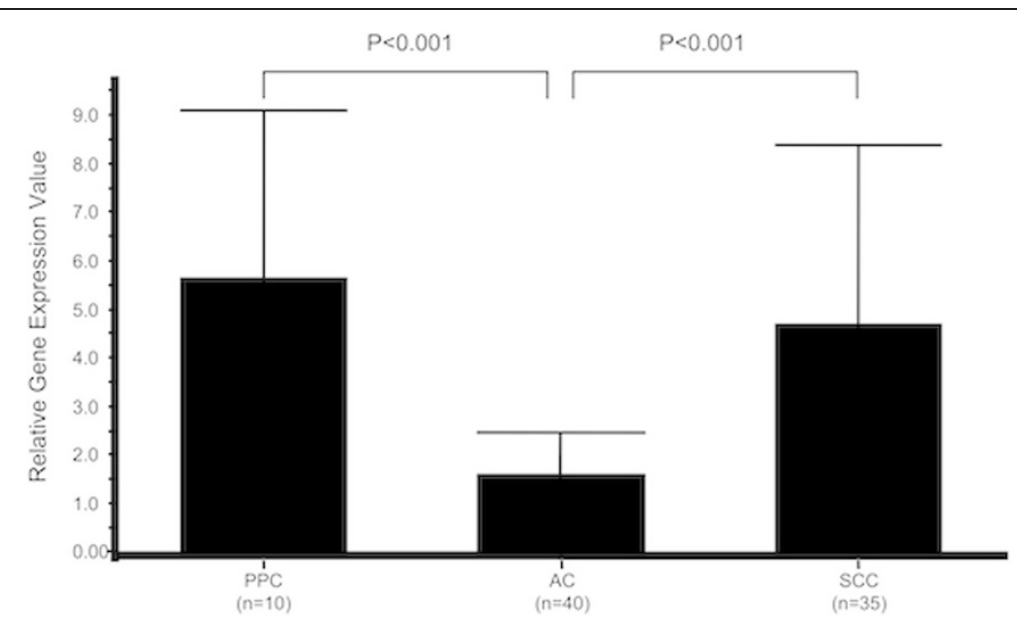

Fig. 1 Comparison of relative gene expression of thymidylate synthase in cancer specimens. PPC, pulmonary pleomorphic carcinoma; AC, adenocarcinoma; SCC, squamous cell carcinoma

have poor prognosis, scant research has been conducted on this tumor. This is the first known investigation of tumor-related gene expression in resected PPC specimens. Our results showed a significant increase in the level of TS mRNA in PPC compared to AC specimens.

The gene expression levels of the nucleotidemetabolism-related enzymes TS, DPD, and TP in PPC were compared to those in stage-matched NSCLC cases listed in our database. Anti-metabolic drugs, such as pemetrexed, gemcitabine, and 5-fluorouracil and its derivatives, are widely used as cancer chemotherapy agents, and their effects include inhibition of TS, as well as incorporation of its metabolites into RNA and DNA. Lower TS activity is thought to be correlated with greater sensitivity to anti-metabolic drugs $[8,9]$. The present findings showed that gene expression levels of TS mRNA in PPC were significantly higher than in AC, whereas those of DPD and TP mRNA in PPC were similar to those in SCC, in contrast to AC.
Generally, AC is thought to be more sensitive to antimetabolic drugs as compared to SCC. Our results indicate that it is unlikely that PPC is sensitive to anti-metabolic drugs, which agrees with clinical experience.

The expression levels of other tumor-related genes were also examined. Unfortunately, we were not able to examine stage-matched AC and SCC specimens, thus used previously reported data (Table 2).

ERCC1 protein is involved in nucleotide excision repair of damaged DNA and determination of ERCC1 mRNA expression may have be clinically useful for cancer treatment, because one of the mechanisms of resistance to platinum chemotherapy drugs is correlated with high ERCC1 activity $[10,11]$. We found that the gene expression level of ERCC1 mRNA in PPC specimens was similar to that reported in NSCLC. However, findings are limited and do not fully reveal the efficacy of platinum-based chemotherapy, of which cisplatin is a likely candidate for PPC treatment.

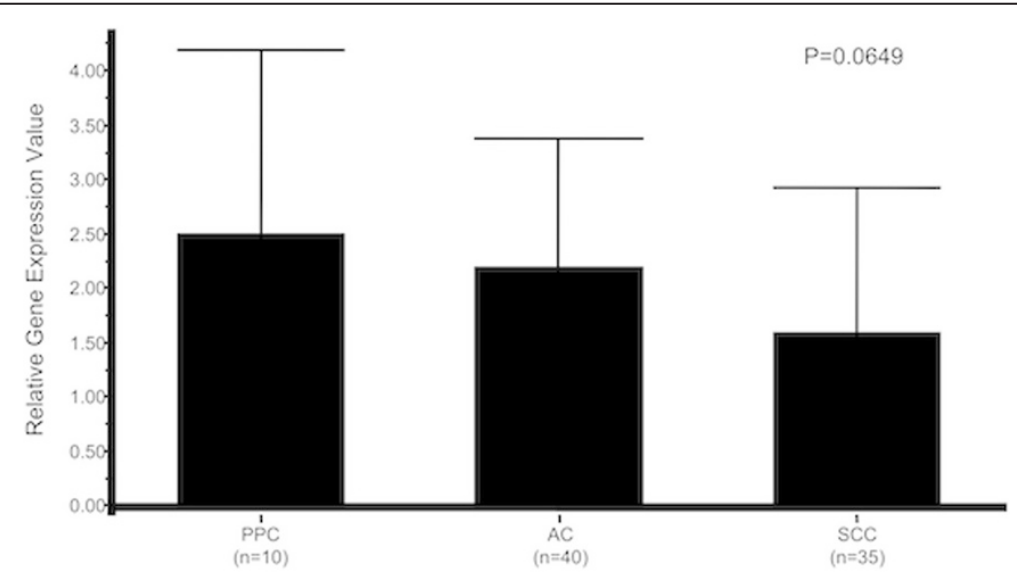

Fig. 2 Comparison of relative gene expression of dihydropyrimidine dehydrogenase in cancer specimens. PPC, pulmonary pleomorphic carcinoma; AC, adenocarcinoma; SCC, squamous cell carcinoma 


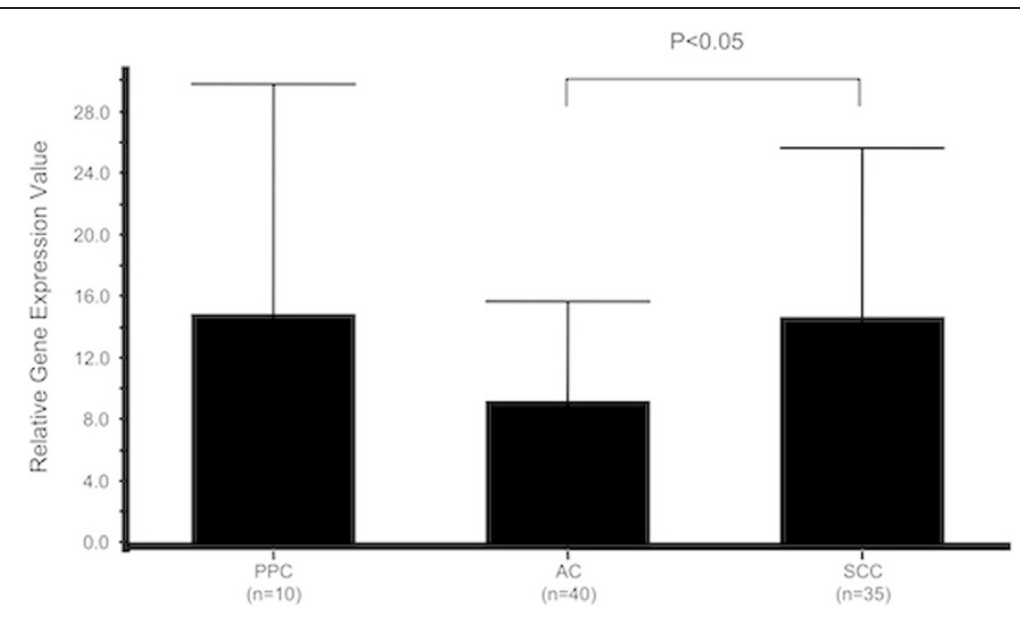

Fig. 3 Comparison of relative gene expression of thymidine phosphorylase in cancer specimens. PPC, pulmonary pleomorphic carcinoma; AC, adenocarcinoma; SCC, squamous cell carcinoma

VEGF is a signal protein that stimulates angiogenesis and anti-VEGF therapies are important for treatment of certain cancers, because solid tumors do not grow beyond a limited size without an adequate blood supply provide by angiogenesis using VEGF signals. VEGF gene expression has been shown to be higher than that of colorectal cancer, thus anti-VEGF therapy may useful for PPC patients. Furthermore, TOPO-1, involved in cell division, is a target of some anti-cancer drugs, such as irinotecan, topotecan, and camptothecin. Nevertheless, there are few reports of a correlation between clinical outcome and TOPO-1 mRNA level determined by the DTP method, though TOPO-1 gene expression was found to be lower than that of thymic cancer.

The present study is limited by its design as an institutional report of a small population, due to the rarity of the disease. Additional studies and case accumulation are necessary.

\section{Conclusion}

Gene expression levels of nucleotide-metabolism-related enzymes in PPC showed a pattern similar to those in SCC, while they were different as compared to AC specimens. Although gene expression of tumor cannot be

Table 2 Mean gene expression values of ERCC1, VEGF, and TOPO1 in PPC and other organ carcinoma specimens by DTP method

\begin{tabular}{lllll}
\hline & & ERCC1 & VEGF & TOPO1 \\
\hline NSCLC [6] & $n=283$ & 1.65 & - & - \\
Colorectal cancer [7] & $n=31$ & - & 3.79 & - \\
Thymic cancer [12] & $n=14$ & 3.62 & 10.06 & 4.02 \\
PPC (present study) & $n=10$ & 1.68 & 8.53 & 2.55 \\
\hline
\end{tabular}

ERCC1 excision repair cross-complementation group 1, VEGF vascular endothelial growth factor, TOPO1 topoisomerase-1, PPC pulmonary pleomorphic carcinoma, DTP Danenberg's tumor profile directly correlated to its sensitivity for anti-cancer drugs, it is unlikely that PPC is sensitive to anti-metabolic drugs. On the other hand, anti-VEGF therapy may be effective for PPC, while cisplatin also remains a viable candidate.

\section{Competing interests}

The authors declare that they have no competing interests.

\section{Authors' contributions}

TO carried out the genetic studies, and drafted the manuscript. YM conceived of the study. SK and TS carried out data collection. HI participated in the design of the study. MC participated in its design and coordination and helped to draft the manuscript. All authors read and approved the final manuscript.

\section{Author details}

'Department of General Thoracic Surgery, Dokkyo Medical University, Mibu 321-0293, Japan. ${ }^{2}$ Department of Cardiovascular and Thoracic Surgery, Dokkyo Koshigaya Hospital, 2-1-50 Minamikoshigaya, Koshigaya, Saitama 343-8555, Japan.

Received: 2 September 2014 Accepted: 28 May 2015 Published online: 03 June 2015

\section{References}

1. Corrin B, Wick MR, Chang YL, Nappi O, Rossi G, Finkelstein SD, et al. Sarcomatoid carcinoma. In: Travis WD et al., editors. Pathology and genetics of tumours of the lung, pleura, thymus and heart, World Health Organization classification of tumours. Lyon: IARC Press; 2004. p. 53-8.

2. Venissac N, Pop D, Lassalle S, Berthier F, Hofman P, Mouroux J. Sarcomatoid lung cancer (spindle/giant cells): an aggressive disease? J Thorac Cardiovasc Surg. 2007;134:619-23.

3. Kawano R, Hino H, Hoshino T, Tagawa K, Yokota T, Ikeda S, et al. Clinicopathological investigation of seven resected cases of pleomorphic carcinoma of the lung, and the results of their chemosensitivity tests. Haigan. 2008;48:106-11.

4. Huang SY, Shen SJ, Li XY. Pulmonary sarcomatoid carcinoma: a clinicopathologic study and prognostic analysis of 51 cases. World J Surg Oncol. 2013;11:252.

5. Olaussen KA, Dunant A, Fouret P, Branmbilla E, Andre F, Haddad V, et al. DNA repair by ERCC1 in non-small-cell lung cancer and cisplatin-based adjuvant chemotherapy. N Eng J Med. 2006;355:983-91.

6. Cobo M, Isla D, Massuti B, Montes A, Sanchez JM, Provencio M, et al. Customizing cisplatin based on quantitative excision repair cross- 
complementing 1 mRNA expression: a phase III trial in non-small-cell lung cancer. J Clin Oncol. 2007;25:2747-54.

7. Kuramochi H, Hayashi K, Uchida K, Miyakura S, Shimizu D, Vallbohmer D, et al. Vascular endothelial growth factor messenger RNA expression level is preserved in liver metastases compared with corresponding primary colorectal cancer. Clin Cancer Res. 2006;12:29-33.

8. Ishihama H, Chida M, Araki O, Karube Y, Seki N, Tamura M, et al. Comparison of 5-fluorouracil-related gene expression levels between adenocarcinomas and squamous cell carcinomas of the lung. Jpn J Clin Oncol. 2009;39:33-6.

9. Salonga D, Danenberg KD, Johnson M, Metzger R, Groshen S, Tsao-Wei DD, et al. Colorectal tumors responding to 5 -fluorouracil have low gene expression levels of dihydropyrimidine dehydrogenase, thymidylate synthase, and thymidine phosphorylase. Clinical Cancer Res. 2000;6:1322-7.

10. Bellmunt J, Paz-Ares L, Cuello M, Cecere FL, Albiol S, Guillem V, et al. Gene expression of ERCC1 as a novel prognostic marker in advanced bladder cancer patients receiving cisplatin-based chemotherapy. Ann Oncol. 2007:18:522-8

11. Lord RV, Brabender J, Gandara D, Alberola V, Camps C, Domine M, et al. Low ERCC1 expression correlates with prolonged survival after cisplatin plus gemcitabine chemotherapy in non-small cell lung cancer. Clinical Cancer Res. 2002;8:2286-91.

12. Karube $\mathrm{Y}$, Ishihama $\mathrm{H}$, Araki O, Seki N, Hayama M, Kobayashi S, et al. Anticancer-drug-related gene expressions in thymic epithelial tumors. Niphon Gekagakkai Zasshi. 2013;114 Suppl 2:730.

\section{Submit your next manuscript to BioMed Central and take full advantage of:}

- Convenient online submission

- Thorough peer review

- No space constraints or color figure charges

- Immediate publication on acceptance

- Inclusion in PubMed, CAS, Scopus and Google Scholar

- Research which is freely available for redistribution 